\title{
Configuring the Infrastructure of Autonomous Logistics' Control Systems
}

\author{
Bernd Scholz-Reiter, Steffen Sowade, and Daniel Rippel \\ BIBA - Bremer Institut für Produktion und Logistik GmbH at the University of Bremen \\ Bremen, Germany \\ bsr@biba.uni-bremen.de,sow@biba.uni-bremen.de,rip@biba.uni-bremen.de
}

\begin{abstract}
Autonomous control is a suitable concept in order to increase the flexibility and robustness of logistic systems by enabling decentralized decision-making and execution at the system elements. However, design of the corresponding control system's infrastructure is complex due to a high number of possible configurations. Further, logistic process experts have to consider several domain specific functional and nonfunctional requirements. Thereto, this paper introduces a three-phase procedure model, which guides logistics experts through the configuration process of the infrastructure of autonomous logistics' control systems. The model employs general design concepts of procedure models and composes them to a new, application area specific model that fits the needs of autonomously controlled logistic systems.
\end{abstract}

Autonomous Logistic Control System, Infrastructure Configuration, Procedure Model

\section{INTRODUCTION}

The efficiency of logistic processes in manufacturing and transportation is crucial for the profitability of a company. Hence, companies employ planning and control systems which manage the achievement of their logistic objectives [1]. Centralized systems generally perform well and are used widely today. However, they lack flexibility and robustness in case of fluctuating capacity supply or demand that is caused by machine breakdowns or rush orders. Hence, researchers investigate alternative concepts, e.g. autonomous controlled logistic processes. Previous research indicates that autonomous logistic processes are able to cope with these fluctuations [2]. Flexibility and robustness is increased by the use of intelligent logistic objects which control their logistic processing locally by themselves [3]. Thereto, the objects are equipped with abilities for rendering and execution of decisions. Realization of these abilities requires additional hard- and software components forming the infrastructure of autonomously working control systems [4]. Logistic process experts have to select and compose suitable components to one infrastructure configuration which considers all scenario-specific aspects. This task is complex, due to a high number of possible infrastructure components.

Thereto, this paper presents a procedure model which structures the infrastructure configuration task for logistics process experts. The remainder of the paper is structured as follows. Section 2 introduces autonomous control in logistics by subsuming its definition, characteristics, and modeling framework. Section 3 defines the term infrastructure and presents an infrastructure layer model which understands infrastructure in a system-theoretic view. Section 4 introduces a new procedure model which guides logistic process experts through the modeling process of the infrastructure of autonomous logistic control systems. Section 5 closes the paper with a conclusion and an outlook to future work.

\section{AutONOMOUS CONTROL IN LOGISTICS}

Autonomous control is one option to cope with increasing complexity and dynamics in logistic systems [2]. The concept employs intelligent logistic objects as system elements which are characterized "by the ability ... to process information, to render and to execute decisions on their own.” [3]. Hülsmann and Windt defined autonomous control in general as "processes of decentralized decisionmaking in heterarchical structures. It presumes interacting elements in non-deterministic systems, which possess the capability and possibility to render decisions independently. The objective of Autonomous Control is the achievement of

\begin{tabular}{|c|c|c|c|c|c|}
\hline System layer & Criteria & \multicolumn{4}{|c|}{ Properities } \\
\hline \multirow{7}{*}{$\begin{array}{l}\text { Decision } \\
\text { system }\end{array}$} & \begin{tabular}{|c|}
$\begin{array}{l}\text { Time behaviour of } \\
\text { objective system }\end{array}$ \\
\end{tabular} & static & mostly static & mostly dynamic & dynamic \\
\hline & $\begin{array}{c}\text { Location of } \\
\text { objective system }\end{array}$ & global & mostly global & mostly local & local \\
\hline & $\begin{array}{c}\text { Organisational } \\
\text { structure }\end{array}$ & hierarchical & $\begin{array}{c}\text { mostly } \\
\text { hierarchical }\end{array}$ & $\begin{array}{c}\text { mostly } \\
\text { heterarchical }\end{array}$ & heterarchical \\
\hline & $\begin{array}{l}\text { Quantity of alter- } \\
\text { native decisions }\end{array}$ & none & some & many & infinite \\
\hline & $\begin{array}{c}\text { Type of decision } \\
\text { making }\end{array}$ & static & \multicolumn{2}{|c|}{ rule-based } & learning \\
\hline & \begin{tabular}{|c|}
$\begin{array}{c}\text { Location of decision } \\
\text { making }\end{array}$ \\
\end{tabular} & system layer & \multicolumn{2}{|c|}{ subsystem layer } & $\begin{array}{c}\begin{array}{c}\text { system-elements } \\
\text { layer }\end{array} \\
\end{array}$ \\
\hline & System behaviour & $\begin{array}{c}\text { elements and } \\
\text { system } \\
\text { deterministic }\end{array}$ & $\begin{array}{c}\text { elements non-/ } \\
\text { system } \\
\text { deterministic }\end{array}$ & $\begin{array}{c}\text { system non-I } \\
\text { elements } \\
\text { deterministic }\end{array}$ & $\begin{array}{l}\text { elements and } \\
\text { system non- } \\
\text { deterministic }\end{array}$ \\
\hline \multirow{3}{*}{$\begin{array}{l}\text { Information } \\
\text { system }\end{array}$} & Data storage & central & mostly central & mostly decentral & decentral \\
\hline & Data processing & central & mostly central & mostly decentral & decentral \\
\hline & Interaction ability & none & data allocation & communication & coordination \\
\hline \multirow{3}{*}{$\begin{array}{l}\text { Execution } \\
\text { system }\end{array}$} & Flexibility & inflexible & less flexible & flexible & highly flexible \\
\hline & Identification ability & $\begin{array}{l}\begin{array}{l}\text { no elements } \\
\text { identifiable }\end{array} \\
\end{array}$ & $\begin{array}{c}\begin{array}{c}\text { some elements } \\
\text { identifiable }\end{array} \\
\end{array}$ & $\begin{array}{c}\begin{array}{c}\text { many elements } \\
\text { identifiable }\end{array} \\
\end{array}$ & $\begin{array}{l}\text { all elements } \\
\text { identifiable }\end{array}$ \\
\hline & Measuring ability & none & others & self & self and others \\
\hline
\end{tabular}

Figure 1. Catalogue of Criteria of Autonomous Control [5]

\begin{tabular}{|c|c|c|c|c|}
\hline System layer & Criteria & \multicolumn{3}{|c|}{ Properties } \\
\hline \multirow{3}{*}{$\begin{array}{l}\text { Systemic } \\
\text { aspects }\end{array}$} & Control representation & virtual object & mixed & real object \\
\hline & Extent of ability transfer & none & some & every \\
\hline & Place of abilities & centralized & mixed & decentralized \\
\hline & & \multicolumn{3}{|c|}{ Increasing level of autonomous control } \\
\hline
\end{tabular}

Figure 2. Systemic Characteristics [4] 
increased robustness and positive emergence of the total system due to distributed and flexible coping with dynamics and complexity." [3]. Hence, presence of decision alternatives is the most important precondition in order to allow local decision making by logistic objects themselves [6]. Further, intelligent logistic objects require decision competence in form of knowledge about methods and algorithms, as well as about environment and object specific data.

\section{A. Characteriziation of Autonomous Control}

Autonomous logistic systems can be characterized by their degree of autonomous control, their degree of complexity, and their degree of logistic objective achievement. Each measure is a vector containing several key indicators. A catalogue of criteria is used in order to characterize autonomous logistic systems by their degree of autonomous control [5]. The catalogue allocates several criteria to three system layers: decision system, information system, and execution system (Fig. 1). Each criterion expresses the single grade of autonomy for this criterion. The dark shaded boxes in Fig. 1 represent one exemplary system instance. The relative importance of each criterion to each other is weighted by a pair-wise comparison. The properties describe the maximum level of autonomous control that a system can achieve. However, specific applications may have a lower level [3].

While the catalogue of criteria presents several criteria in three different system layers, it neglects systemic aspects of a system at a glance. Systemic aspects are useful in order to characterize different control system architectures, as well as logistic objects and their infrastructure components (Fig. 2). First, essential abilities can be placed at different locations, either centralized or rather decentralized. Second, the extent of ability transfers varies between none, some, or every ability. The higher the degree of autonomous control increases, the more abilities are transferred to the system elements. Third, either virtual logistic objects located in a (semi-) centralized computer system or real logistic objects are able to execute control methods. In addition, control methods can be performed by native system elements or by elements additionally placed in the system [4].

Logistic systems are complex systems which embody several interrelated system elements and interact with their system external environment. Windt et al. distinguish three different categories of complexity and compose them to one complexity vector in order to compare the complexity of different logistic systems. Organizational complexity emerges from the number and diverseness of system elements and process flows. Time-related complexity refers to changes of system elements and process flows in progress of time as well as to a snapshot at a specific point in time or period of time. Systemic complexity distinguishes system elements and process flows with regard to the system boarder as complexity that is induced internal or external [7].

In order to determine the degree of logistic objective achievement for a specific system, a measurement system as well as a calculation method is required. Windt et al. propose a method for the measurement of basic logistic objectives and for a successive calculation of the degree of logistic objective achievement. According to Wind et al., a logistic

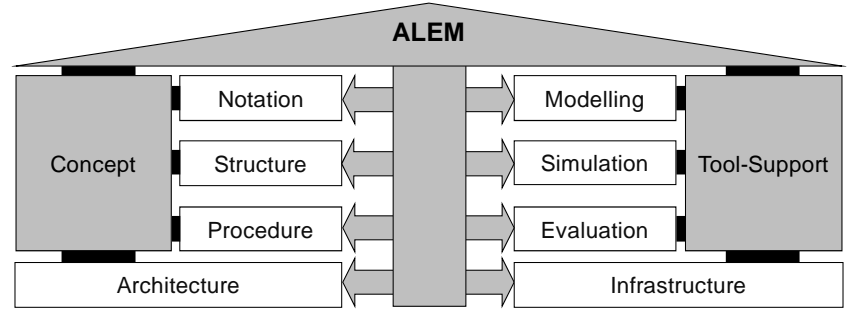

Figure 3. Autonomous Logistics Engineering Methodology [8]

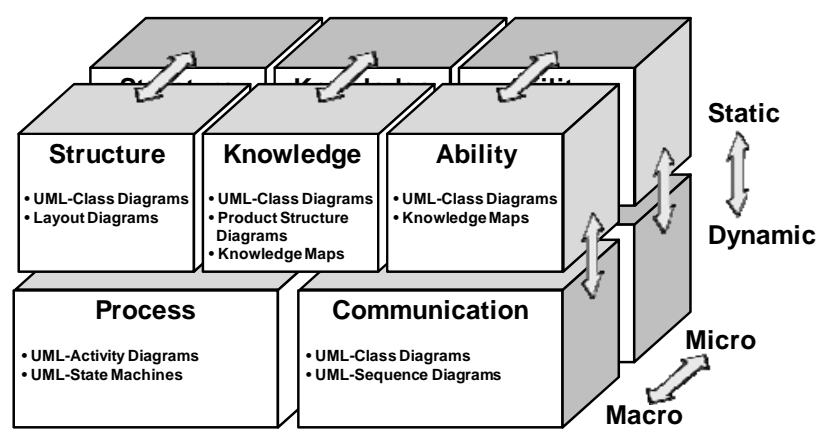

Figure 4. ALEM View Concept [8]

system measures specific objectives, e.g. utilization, for each logistic object and calculates the relative difference to the planned achievement of objectives. The result is stored in an unweighted vector of the relative objective achievement per object. Then each objective component of this vector is weighted by its relative importance. The resulting vector describes the weighted relative objective achievement for one object. Due to several reasons, the importance of logistic objects in a system may differ. Hence, a pair wise comparison of all objects is proposed in order to determine the individual importance of each logistic object. Finally, the degree of logistic objective achievement of the overall system is calculated as sum of the object-weighted individual objective achievement divided by the sum of all objects weighting factors [9].

\section{B. Modeling Autonomuos Contolled Processes}

In order to enable logistic process experts to model autonomous logistic business processes the Autonomous Logistics Engineering Methodology (ALEM) is developed by researchers of the Collaborative Research Centre (CRC) 637 [10], [11]. ALEM comprises a notation, a structuring view concept, and a procedure model. Further, the ALEM design process relies on decisions about a system's architecture and on selection of infrastructure components that are specific for autonomous controlled logistic processes. ALEM integrates all methods into a software tool (ALEM-T) which guides logistic process experts through the process of model creation, simulation, and evaluation (Fig. 3).

ALEM's notation bases on the Unified Modeling Language (UML) and extends it by diagrams that are specific for the domain of autonomous logistic processes. For example, knowledge maps, a layout diagram, and product structure diagrams have been added [11], [13]. 


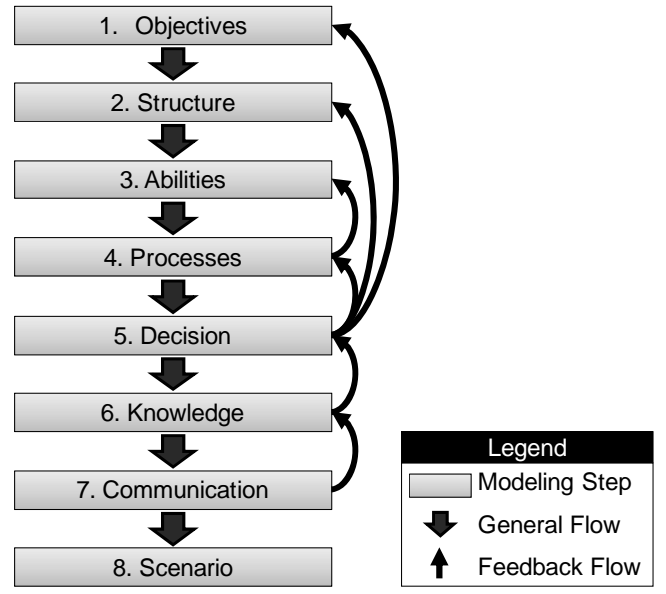

Figure 5. ALEM Procedure Model [11]

ALEM applies a view concept in order to deal with the complexity of its models [6], [14]. Each view focuses on single aspects of the overall model and enables designing lower complex model segments [15]. ALEM uses five primary views in order to divide the overall model into distinct, semantic aspects (Fig. 4). In addition, the views group model segments in static and dynamic aspects. Static aspects describe time invariant model components. Dynamic aspects subsume time depending procedures performed by logistic objects. Further, model segments are differentiated into micro aspects, concerning an object's internal model and macro aspects, e.g. describing the overall systems structure.

The view concept differentiates model segments for an ALEM model's structure, knowledge, abilities, processes, and communication. Each view uses multiple diagrams to depict a certain aspect [8]. The structure view is a static view, primarily containing macro aspects. It defines all logistic objects present in a system and their relationships. In addition, this view includes the spatial layout of a modeled scenario. The knowledge view is a part of the static view and mainly contains micro aspects. It describes all knowledge of an object, e.g. its objectives. UML-Class diagrams represent the logistic object's knowledge in form of attributes. Moreover, the knowledge view employs specialized diagrams, like product structure diagrams and knowledge maps. The ability view is a static view and includes micro as well as macro aspects. It uses UML-Class diagrams in order to represent abilities of logistic objects. Further, the ability view employs knowledge maps to assign abilities to specific logistic objects. The process view is a part of the dynamic view and incorporates micro and macro aspects. It uses UML-State Machine and UML-Activity diagrams in order to describe the behavior of logistic objects. The communication view is dynamic and mainly contains macro aspects. It employs UML-Class and UML-Sequence diagrams. Class diagrams define messages exchanged between logistic objects, while sequence diagrams represent communication protocols.

Kolditz proposes the procedure model ALEM-P (procedure) in order to guide logistic process experts through the modeling process of autonomous logistic business processes (Fig. 5). ALEM-P consists of eight steps, each dealing with one specific aspect of autonomous logistic business processes. Several aspects are worth to be mentioned in detail. However, for a full description of each step see [11].

Although the procedure model's steps show a straight sequence, the modeling order can vary in certain cases. Reordering of the procedure steps depends on whether a topdown approach or a bottom up approach is used for modeling. For instance, if a specific method or algorithm for autonomous decision making shall be employed, the decision process has to be described before modeling an object's abilities. ALEM-P allows feedback loops within the modeling process in order to include new aspects of a system when they appear while modeling.

Objectives are modeled in the first step and are a precise kind of knowledge that is allocated at a logistic object. Step four and five belong to the process model, but refer to different views. Decisions depict on the micro view of a decision process itself, while processes are elements of the macro view and describe sequences of tasks of logistic objects. The last procedure step is used in order to picture a concrete scenario of a system. A scenario is an overall instance model of all ALEM submodels described in the seven previous steps. It shows the spatial configuration of all system elements at a certain point in time, e.g. when initiated. During the last step all scenario parameters values are named, e.g. the terminal diagram of a machine. However, ALEM-P lacks to describe an exact procedure for the configuration of the infrastructure of autonomous logistic processes.

\section{INFRASTRUCTURE MODEL}

The historic outline of the term infrastructure shows an ongoing adaption from a strict technical meaning to military and economic use and towards its application in politics and informatics. In origin infrastructure is a technical term and denotes durable facilities which are connected to the ground [16]. It is also used in deregulation politics for transportservice-oriented basic works in telecommunication, electricity, gas, and water supply. Further, informatics names hardware and software equipment as infrastructure that provides a set of information technology services to users. Today and with respect to its historical use, infrastructure is often understood as economical-organizational foundation. Instead, Klaus defines infrastructure as: "the elementary human-made facilities, which are a precondition for a high developed economy and may change over time. Its main characteristics are its base-character, artificiality, indispensability for proper functionality, and changeability.” [16].

Although Klaus' definition is much more general than others, neither it converges with the other definition approaches to a unique general understanding of infrastructure, nor does it includes specifics of logistic systems. For this reason, Scholz-Reiter et al. define more abstract and with a systemtheoretic view: "Infrastructure includes all system elements which are placed artificially into a given system, called native system. These system elements must be essential to enable specific higher order services within the system by use of capabilities supplied by native system elements and by artificially inserted system elements. " [4]. 


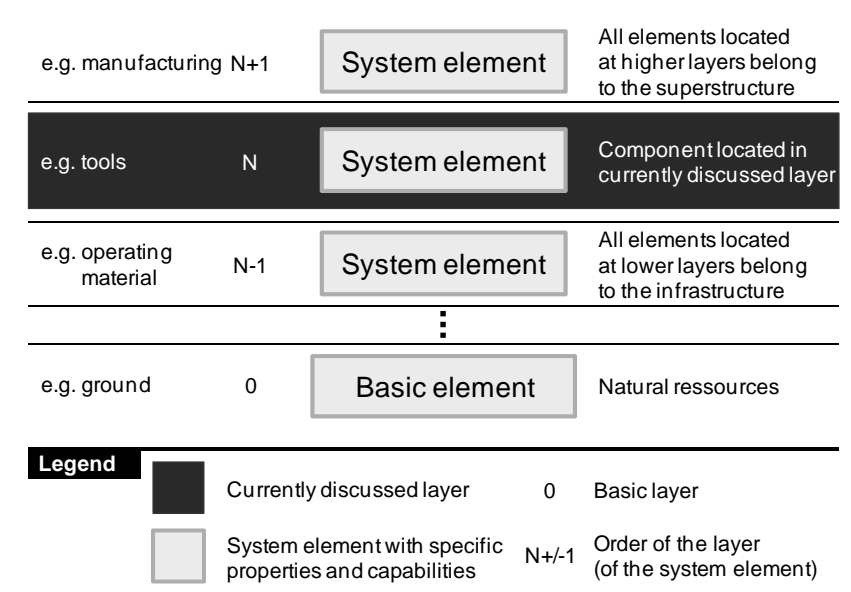

Figure 7. Infrastructure Layer Model [4]

Neither elements of a native system nor additional system elements alone are able to perform specific demanded activities. Thus, artificially inserted elements are a precondition in order to provide specific functions or, respectively, to carry out higher order tasks. For instance, operation of a transportation service in a spatial delimited logistic system requires natural ground and artificially tracks. Further, the definition of Scholz-Reiter et al. depicts a hierarchy of system elements which can be used in order to derive a generic infrastructure layer model (Fig. 6). This new model bases on distinct layers whose elements provide specific functional services to higher layer elements. For instance, all system elements located in layer $\mathrm{N}-1$ are infrastructure from the viewpoint of layer $\mathrm{N}$. At the bottom, the model shows elements of the corresponding native system.

The main characteristics of infrastructure are its basecharacter, artificially integrated elements, its indispensability for proper functionality, and the changeability of infrastructure components [16]. Establishment of infrastructure requires resources, i.e. space, and causes sunk costs. The sociotechnological development determines its social impact. Contrary to Klaus, the artificiality refers to the process to add another element, but not necessarily to the type of element itself [16]. For instance, a wooden pier at a lake shore is made of national grown lumber. However, it does not belong naturally to a shore. The piers structure is artificial as well as the process of its creation and placement.

Authors with a background in deregulation politics often classify infrastructure by its dedication, usage, materiality, network orientation, and level type [16], [17]. Table 1 presents these characteristics with their corresponding values. The last three rows are partly counterintuitive and need further explanation. Contrary to [16], [17], the term

TABLE I. INFRASTRUCTURE CLASSIFICATION [15]

\begin{tabular}{|l|c|c|}
\hline \multicolumn{1}{|c|}{ Characteristic } & \multicolumn{2}{c|}{ Value } \\
\hline Dedication & Public & Private \\
\hline Usage & Productive & Consumptive \\
\hline Materiality & Material & Immaterial \\
\hline Network Orientation & Network-based & Non-network-based \\
\hline Level Type & Primary & Secondary \\
\hline
\end{tabular}

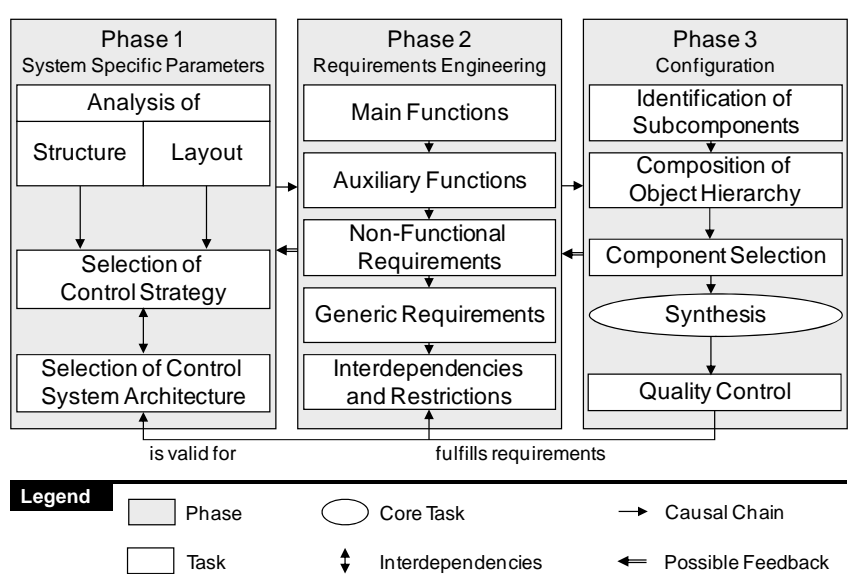

Figure 6. Infrastructure Configuration Prodecure Model

immaterial is used instead of institutional/personal. Both describe an immaterial regulation framework and the capabilities of a population, respectively. However, the latter classification is incomplete and imprecise from an engineering science viewpoint, because it neglects technical norms and standards. Thus, the term immaterial is employed. Further, an infrastructure is network-based, if its elements form a network which enables the system's functionality. Such networks are characterized by the presence of nodes being interconnected via links. The links usually transport data, energy, or physical goods from one node to another. Finally, secondary infrastructure requires subordinated infrastructure; primary infrastructure does not. System elements located in layer $0+1$ are primary infrastructure, while higher layer system elements are secondary infrastructure and so on [4].

\section{INFRASTRUCTURE CONFIGURATION PROCEDURE}

The previous section outlined the concept of autonomous control in logistics, depicted a layer model in order to visualize the term infrastructure, and introduced different views on system architectures and their elements in case of autonomously controlled logistic processes. Looking closer, there exist several possible infrastructure components. The task to arrange these components to a valid configuration is complex and difficult for logistic process experts. Hence, this section introduces a procedure model for the configuration of the infrastructure of autonomous logistics' control systems. The first subsection describes the procedure model at a glance, while subsequent subsections provide more details of this new procedure model.

\section{A. Model Overview}

The new developed procedure model for the configuration of the infrastructure of autonomous logistics control systems (ALEM-C; configuration procedure model) employs three phases each subsuming four to five tasks (Fig. 7). It follows an iterative approach and uses feedback from succeeding phases in order to create new configuration increments. The sequence of a phase's tasks is a recommendation that a logistic expert can alter on demand. Moreover, the configuration process is driven by knowledge about a logistic process described in an ALEM model, by derived 
functional and non-functional requirements, as well as by domain specific knowledge about classes of technologies and components, instances of them, and their parameters. Hence, ALEM-C employs ALEM submodels, like structure and interaction models. Further, it extends the existing ALEM procedure model (ALEM-P) with specific elements of an infrastructure configuration process.

\section{B. Phases of the Configuration Procedure Model}

In the first phase, a logistic expert gathers and cumulates system specific parameters, which affect the latter syntheses of infrastructure components. He specifies and analyzes structure, layout, and control strategy of a planned logistic system, as well as the control system's micro and macro architecture. The second phase is used to identify all functional, non-functional, and generic requirements of the infrastructure based on an ALEM-model of the intended logistic system. The requirements are derived from and are associated with functions of the ALEM process and interaction submodels. At the end of the second phase, a logistic expert determines interdependencies and restrictions which result from an analysis of the requirements. Configuration of a control system's infrastructure components takes place in the third phase. In its first two tasks, infrastructure subcomponents are identified and clearly arranged in an object hierarchy. Third, a logistic expert selects technical components for each functional class of the control system whose attributes fit the demanded requirements. Then he performs the core task and synthesizes all components to one configuration increment. Finally, a configurations quality is checked.

\section{Quality Management Mechanisms}

Each increment faces a quality control task at the end the third phase. A built-in quality gate analyses, if the current configuration increment fits the system specific parameters and if it fulfills the requirements determined earlier. The assessment's results provide feedback for the development of successive increments. The configuration process iterates until an adequate solution is verified by the quality gate in order to ensure a feasible and economically solution. While the final quality control task is a major task and is located in the third phase of the procedure model, there exist mechanisms for quality management within each task too. These secondary mechanisms enable logistic process experts to verify the syntactical correctness and the completeness of each tasks outcome. Due to the relation between an ALEM model and the derived functional and non-functional requirements, every requirement has to be linked to a class in the ALEM model and vice versa. Otherwise the infrastructure model would be incomplete and enforcing logistic process experts to add requirements of ALEM model elements.

\section{Iteration Mechanisms}

The procedure model employs two different iteration mechanisms. The first mechanism is repetitive, the second is recursive. The quality gate task performed in the third phase triggers the repetitive mechanism. It systematically points out deviations between the infrastructure specification, the general conditions, and the requirements defined earlier.
These deviations constitute feedback for previous phases. Feedback sent to the first phase usually requires adjustments of structural parts and design decisions in ALEM-models, while feedback addressed to the second phase demands modification of the employed requirements model. Thus, logistic experts repeat the tasks of the corresponding phases and process all following phases again. Second, the repetitive mechanism employs recursion in order to describe infrastructure subcomponents in detail. A logistic expert is allowed to repeat previous tasks of a phase within this specific phase in order to integrate new details and insights into the infrastructure model and its components. Although iteration of specific tasks or phases is time-consuming, it is a proper technique to increase the quality of a model.

\section{CONCLUSION AND OUTLOOK}

This paper presented autonomous logistic systems as one application area of embedded systems. In both cases small units of hardware and software are combined and integrated into a larger system in order to perform specific tasks for and within this surrounding system. Further, this paper introduced the new knowledge-driven procedure model, ALEM-C (configuration procedure model), which assists logistic process experts during the configuration process of the infrastructure of autonomous logistics' control systems. ALEM-C employs three phases in order to collect system specific parameters, to derive requirements for infrastructure components, and to synthesize a configuration increment. The model follows an iterative, repetitive and recursive, approach and allows changing of the sequence of a phase's tasks. ALEM-C employs ALEM submodels and extends the existing ALEM procedure model (ALEM-P) with specific elements of an infrastructure configuration process.

The authors plan to enhance the new procedure model with a methodical guideline which specifies a set of suitable, exemplary methods and their method class for each task. Further, the detailed configuration model, ALEM-C, is going to be tested with help of logistics' scenarios acquired with project partners. In addition, the authors plan to development a data base which stores clusters of different technologies' properties. In the far future, the authors plan to perform future research in the subject area of cost-benefit-models for autonomous logistic business processes.

\section{ACKNOWLEDGMENT}

This research is funded by the German Research Foundation (DFG) as part of the Subproject B2 of the Collaborative Research Center 637 "Autonomous Cooperation Logistic Processes - A Paradigm Shift and Its Limitations” (CRC 637).

\section{REFERENCES}

[1] Luczak, H., Eversheim, W., Schotten, M., 2001, Pro-duktionsplanung und -steuerung: Grundlagen, Ge-staltung, Konzepte, Springer Verlag, Berlin

[2] B. Scholz-Reiter, H. Rekersbrink, M. Görges, "Dynamic Flexible Flow Shop Problems - Scheduling Heuristics vs. Autonomous Control," in CIRP Annals - Manufacturing Technology, vol. 59, no. 1, pp. 465-468, 2010. 
[3] M. Hülsmann and K. Windt, Eds., Understanding of Autonomous Cooperation and Control in Logistics - The Impact of Autonomy on Management, Information, Communication and Material Flow. Berlin: Springer Verlag, 2007.

[4] B. Scholz-Reiter, St. Sowade, and D. Rippel, "Modeling the Infrastructure of Autonomous Logistic Control Systems," in European Conference of Systems (ECS'10), V. Mladenov, K. Psarris, N. Mastorakis, A. Caballero, and G. Vachtsevanos, Eds. Puerto de la Cruz, Tenerife: WSEAS Press, 2010, pp. 295-300.

[5] F. Böse and K. Windt, "Catalogue of Criteria for Autonomous Control," in Understanding Autonomous Cooperation and Control in Logistics - The Impact of Autonomy on Management, Information and Communication and Material Flow, M. Hülsmann and K. Windt, Eds. Berlin: Springer, 2007, pp. 57-72.

[6] B. Scholz-Reiter, H. Höhns, J. Kolditz, and T. Hildebrandt, “Autonomous Supply Net Coordination,” in Proceedings of 38th CIRP Manufacturing Systems Seminar, Florianopolis, 2005.

[7] K. Windt, T. Philipp, and F. Böse, "Complexity Cube for the Characterization of Complex Production Systems,” in International Journal of Computer Integrated Manufacturing - Digital Enterprise Technology: Perspectives and Future Challenges, London: Taylor \& Francis, vol. 21, no. 2, pp. 195-200, 2008.

[8] B. Scholz-Reiter, D. Rippel, and St. Sowade, "Modeling and Simulation of Autonomous Logistic Processes," in NAUN European Conference of Control (ECC'10) , V. Mladenov, K. Psarris, N. Mastorakis, A. Caballero, and G. Vachtsevanos, Eds. Puerto de la Cruz, Tenerife: WSEAS Press, 2010, pp. 148-153.
[9] K. Windt, T. Philipp, F. Böse, and T. Becker, „Application of a threecomponent evaluation system for autonomous control in logistics," in Proceedings of the Institution of Mechanical Engineers, Part B: Journal of Engineering Manufacture, London: Sage Publications, vol. 224, no. 8, pp.1267-1276, 2010.

[10] B. Scholz-Reiter, J. Kolditz, and T. Hildebrandt, "Engineering autonomously controlled logistic systems," in International Journal of Production Research, vol. 47, no. 6, pp. 1449-1468, 2009.

[11] J. Kolditz, "Vorgehensmodell zur Erstellung von Fachkonzepten für selbststeuernde produktionslogistische Prozesse,“ in Informationstechnische Systeme und Organisation von Produktion und Logistik, B. Scholz-Reiter, Eds. Berlin, Germany, GITO Verlag, 2009

[12] B. Scholz-Reiter, St. Sowade, D. Rippel, M. Teucke, M. Özsahin, and T. Hildebrandt, "A Contribution to the Application of Autonomous Control in Manufacturing," International Journal of Computers, vol. 3, no. 3, pp. 279-291, 2009.

[13] B. Scholz-Reiter, J. Kolditz, and T. Hildebrandt, "UML as a Basis to Model Autonomous Production Systems," in Digital Enterprise Technology: Perspectives and Future Challenges, P. F. Cunha and P. Maropoulos, Eds. Berlin: Springer Verlag, 2007, pp. 553-560.

[14] A.-W. Scheer, Business Process Engineering - Reference Models for Industrial Enterprises. Telos: Springer, 1994.

[15] A.-W. Scheer, ARIS - Modellierungsmethoden, Metamodelle, Anwendungen, 4th, Ed. Berlin: Springer Verlag, 2001.

[16] S. Klaus, "Deregulierung der netzbasierten Infrastruktur," Ph.D. dissertation, Zürich: Universität Zürich, 2009.

[17] R. Jochimsen, "Theorie der Infrastruktur : Grundlagen der marktwirtschaftlichen Entwicklung,“ Ph.D. dissertation, Tübingen: Mohr, 1966. 